\title{
Actualización en el manejo nutricional de la insuficiencia pancreática exocrina: revisión de la literatura
}

\author{
Update on the nutritional management of exocrine pancreatic \\ insufficiency: A literature review \\ Atualização sobre o manejo nutricional da insuficiência pancreática \\ exócrina: revisão da literatura
}

Marisa Canicoba 1", Lucía De Nobili', Anabel Villagra²

Recibido: 9 de febrero de 2021. Aceptado para publicación: 11 de marzo de 2021.

Publicado en línea: 18 de marzo de 2021.

https://doi.org/10.35454/rncm.v4n3.282

\section{Resumen}

La insuficiencia pancreática exocrina (IPE) produce mala digestión y malabsorción de nutrientes, lo que impacta negativamente en el estado nutricional. Si no se trata adecuadamente, esta conduce a una pérdida de peso progresiva y posibles deficiencias nutricionales, lo que aumenta la morbimortalidad de los pacientes, las reinternaciones y los costos sanitarios. La detección y el tratamiento oportuno de la IPE afectan positivamente el pronóstico de la enfermedad. El cuidado nutricional, el cual consta de un proceso continuo, tiene que formar parte de la atención integral del paciente, por tanto, debe ser un componente inherente a su cuidado. La detección temprana de pacientes en riesgo de desnutrición, o desnutridos, permite una intervención nutricional precoz, con el propósito de atenuar el impacto nutricional de la IPE. El objetivo de esta revisión es proporcionar evidencia científica acerca de la IPE y brindar un enfoque práctico para el manejo nutricional y el tratamiento clínico de la misma, importante por su impacto en la morbimortalidad y la calidad de vida.

Palabras clave: insuficiencia pancreática exocrina, evaluación nutricional, terapia de reemplazo enzimático pancreático, terapia nutricional.

\section{Summary}

Exocrine pancreatic insufficiency (EPI) results in maldigestion and malabsorption, with a negative impact on the patient's nutritional status. If not treated adequately, it leads to a progressive loss of weight and potential nutritional deficiencies, increasing morbidity and mortality, hospital readmissions and healthcare costs. Detection and appropriate treatment of EPI has a positive effect on disease prognosis.

Nutritional care as a continuous process has to be part of the comprehensive approach to the patient and, therefore, an inherent component of care.

Early detection of patients at risk of malnutrition or already malnourished allows for early nutritional intervention and mitigation of the nutritional impact of EPI.

The objective of this review is to offer scientific evidence on EPI and to provide a practical approach to nutritional management and clinical treatment of this condition.

Keywords: Exocrine Pancreatic Insufficiency; Nutritional Assessment; Pancreatic Enzymes Replacement Therapy; Nutritional Therapy.

\section{Resumo}

A insuficiência pancreática exócrina (IPE) produz má digestão e má absorção de nutrientes, impactando negativamente no estado nutricional. Quando não è tratada adequadamente, leva à perda progressiva de peso e possíveis deficiências nutricionais, aumentando a morbimortalidade dos pacientes, as re-hospitalizações e os custos em saúde. A detecção e o tratamento precoce da IPE afetam positivamente o prognóstico da doença. $O$ cuidado nutricional, que consiste num processo contínuo, deve fazer parte da atenção integral do paciente e, portanto, deve ser um componente inerente ao seu cuidado. A detecção precoce de pacientes em risco de desnutrição ou desnutridos, permite uma intervenção nutricional antecipada, com o objetivo de atenuar o impacto nutricional da IPE. O objetivo desta revisão é proporcionar evidências científicas sobre a IPE e fornecer uma abordagem prática para o manejo nutricional e o tratamento clínico da doença, importante por seu impacto na morbimortalidade e na qualidade de vida.

Palavras-chave: insuficiência pancreática exócrina, avaliação nutricional, terapia de reposição enzimática pancreática, terapia nutricional. 
Departamento de Alimentación y Dietoterapia. Hospital Nacional Alejandro Posadas, Argentina.

2 Servicio de Nutrición. Hospital de Alta Complejidad El Cruce, Argentina.
'Correspondencia: Marisa Canicoba. marisa.canicoba@gmail.com

\section{INTRODUCCIÓN}

La insuficiencia pancreática exocrina (IPE) se define como una reducción en la cantidad o la actividad de las enzimas pancreáticas a un nivel que resulta insuficiente para mantener los procesos digestivos normales ${ }^{(1-3)}$. Las causas de la IPE son numerosas, esta puede desarrollarse en pacientes con enfermedades pancreáticas o extrapancreáticas ${ }^{(2)}$.

Inicialmente puede presentarse con síntomas de mala digestión, malabsorción y deficiencias nutricionales, lo que aumenta la morbimortalidad ${ }^{(3)}$. El diagnóstico de la IPE requiere de la evaluación combinada de los síntomas relacionados con mala digestión, parámetros nutricionales y una prueba de función pancreática no invasiva, aunque en la práctica clínica se ve obstaculizado por la falta de pruebas precisas ${ }^{(4)}$.

El tratamiento de la IPE se basa en un plan de alimentación saludable, no restrictivo, junto con la terapia de reemplazo enzimático pancreático (TREP). La dosis de enzimas pancreáticas orales se debe individualizar en función de la respuesta sintomática, de los hábitos alimentarios de cada paciente y de la evaluación objetiva del estado nutricional ${ }^{(3)}$.

Esta revisión proporciona evidencia relacionada con la prevalencia, etiología, diagnóstico y tratamiento de la IPE, basada en una búsqueda en la literatura, complementada con información adicional del conocimiento personal y clínico de la experiencia de los autores. El reconocimiento y tratamiento de la IPE ayuda a evitar la morbilidad y mortalidad, junto con el deterioro de la calidad de vida.

\section{MÉTODOS}

\section{Estrategias de búsqueda}

Se realizó una búsqueda bibliográfica a través de MEDLINE (PubMed) y Cochrane Library de ensayos controlados. Se utilizaron los siguientes términos de búsqueda en todas las combinaciones posibles: IPE, TREP, pancreatitis aguda (PA), pancreatitis crónica $(\mathrm{PC})$, cáncer de páncreas $(\mathrm{CP})$.

Se incluyeron artículos que cumplían con los siguientes criterios de inclusión para estudios en humanos:
- Revistas en inglés.

- Estudios de diseño asignados al azar, revisiones sistemáticas, guías de práctica clínica, estudios aleatorizados controlados en adultos ( $\geq 18$ años).

- Informe clínico con resultados de interés, que evaluaron la IPE en los resultados de eventos clínicos.

Se examinaron en forma independiente todos los estudios identificados y se realizó la inclusión de aquellos que se consideraron de interés. En la Figura 1 se puede observar la selección de artículos.

\section{IPE: DEFINICIÓN, ETIOLOGÍA, PRESENTACIÓN CLÍNICA Y FISIOPATOLOGÍA}

La IPE se define como el estado de mala digestión de macro y micronutrientes causado por la secreción insuficiente de enzimas pancreáticas o bicarbonato de sodio, debido a la pérdida del parénquima o a la obstrucción del conducto pancreático principal o por causas que producen una inadecuada activación enzimática o sobreproducción de ácido gástrico o una baja estimulación neurohormonal ${ }^{(2,5,6)}$.

Si la IPE no se diagnostica ni se trata, esta conduce al desarrollo de desnutrición (DN) calórica-proteica, deficiencias nutricionales de micronutrientes, vitaminas liposolubles (A, D, E, K) y proteínas plasmáticas (albúmina, prealbúmina, proteína fijadora de retinol, apolipoproteínas, lipoproteínas de alta densidad y transferrina $)^{(2,4,5,7-11)}$.

Por dichas deficiencias, la IPE se asocia de forma independiente y significativa con el desarrollo de:

- Osteopenia y osteoporosis: constituyen patologías frecuentes y subestimadas en pacientes con $\mathrm{PC}^{(11)}$. Según la revisión sistemática y metaanálisis realizado por Duggan y colaboradores, se demostró que 1 de cada 4 pacientes con PC tiene osteoporosis y dos tercios presenta osteoporosis u osteopenia ${ }^{(12)}$.

- Eventos cardiovasculares (ECV): existe una asociación estadísticamente significativa entre la IPE $\mathrm{y}$ el riesgo de $\mathrm{ECV}$ en pacientes con $\mathrm{PC}$, junto con los principales factores de riesgo $\mathrm{CV}$ como el tabaquismo (TBQ) y la hipertensión. En una cohorte 


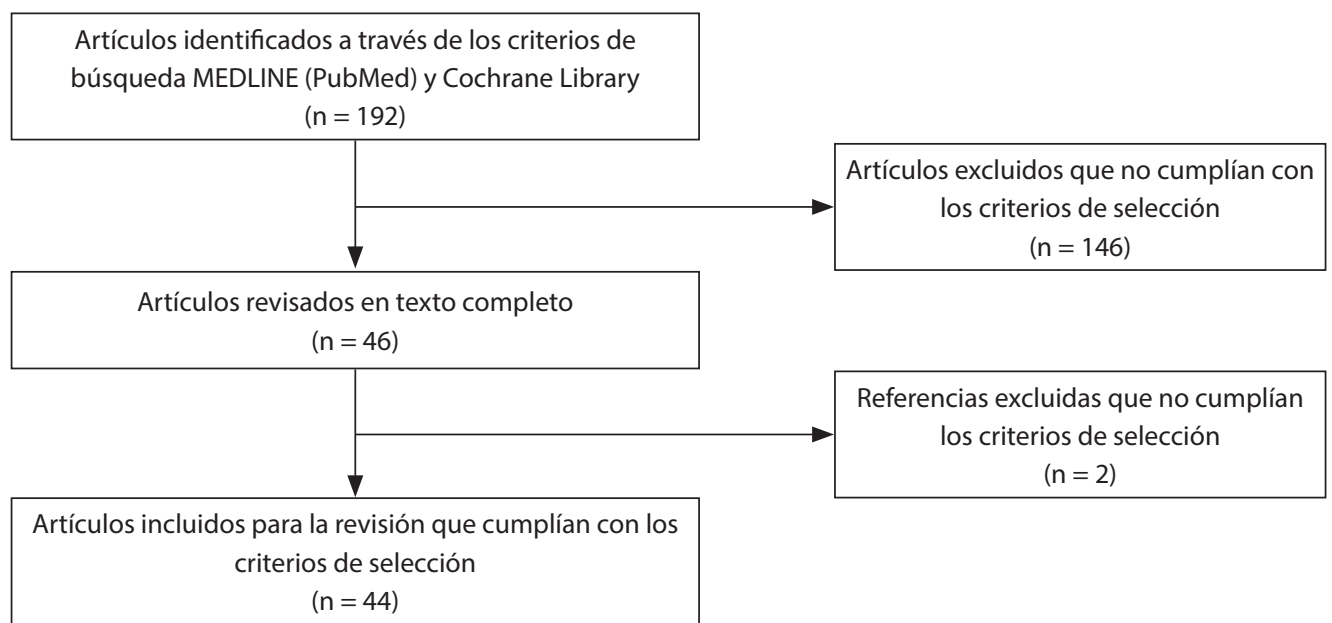

Figura 1. Diagrama de flujo del proceso de selección de los estudios. Fuente: elaboración propia.

de 430 pacientes con PC, De la Iglesia y colaboradores detectaron un índice de tasa de incidencia de ECV de 3,67 (IC $95 \%$ 1,92-7,24; $P<0,001$ ), en los pacientes que tenían IPE ${ }^{(13)}$.

- Sarcopenia: Shintakuya y colaboradores reportaron una asociación independiente y significativa entre IPE y sarcopenia en una serie de 132 pacientes con diferentes enfermedades pancreáticas ${ }^{(14)}$.

En cuanto a su presentación clínica, los síntomas de IPE pueden ser variables. Los más típicos son dolor abdominal, distensión, diarrea, esteatorrea, calambres, flatulencia y pérdida de peso. Para evitar dichos síntomas, los pacientes adaptan la alimentación y, como consecuencia, la pérdida de peso puede ser tardía ${ }^{(1,4,15)}$. No todos los pacientes presentan los síntomas mencionados, por lo cual es mandatorio investigar este trastorno aún en los asintomáticos, en todos los escenarios en los cuales la IPE sea factible ${ }^{(2)}$.

Los síntomas clínicos de la IPE no suelen manifestarse hasta que los niveles de lipasa duodenal caen por debajo del $5 \%-10 \%$ del valor posprandial normal ${ }^{(8)}$. El reconocimiento y tratamiento de la IPE ayuda a evitar la morbilidad y mortalidad, junto con el deterioro de la calidad de vida ${ }^{(6,7)}$.

\section{PREVALENCIA DE IPE}

La prevalencia actual de IPE es muy variable y puede estar asociada con causas pancreáticas o extrapancreáticas (Tabla 1).

\section{Causas pancreáticas}

\section{Pancreatitis crónica}

Es una de las causas más frecuente de IPE. Su prevalencia es del $60 \%$ al $90 \%$, y se hace presente dentro de los 10 a 12 años luego del diagnóstico ${ }^{(16)}$. Los factores que se asocian con el desarrollo de IPE son la duración de la enfermedad, la etiología alcohólica, la presencia de calcificaciones y la obstrucción ductal ${ }^{(21)}$.

El desarrollo de IPE en PC se debe a cambios parenquimatosos o ductales que ocurren por la inflamación, la fibrosis y sus consecuencias ${ }^{(2)}$. La secreción reducida de enzimas pancreáticas altera la digestión de los triglicéridos, lo que provoca una absorción reducida de grasas y vitaminas liposolubles, mientras que la disminución de las proteasas puede provocar DN proteica y deficiencia de vitamina $\mathrm{B}_{12}{ }^{(26)}$. La IPE puede aparecer en cualquier etapa de la enfermedad, y a menudo es subdiagnosticada, por tanto, no es tratada.

\section{Pancreatitis aguda}

La frecuencia de IPE pos-PA es variable debido a la heterogeneidad de los estudios, esta oscila entre $0 \%$ y $83 \%{ }^{(2)}$. Su desarrollo y evolución se relaciona con la etiología de la pancreatitis, la gravedad del episodio, la presencia de necrosis glandular, su localización y extensión (mayor de $50 \%$ ), la necrosis encapsulada (WON [walled off necrosis]), el requerimiento de tratamiento endoscópico, percutáneo o quirúrgico de la necrosis infectada, la recurrencia de la pancreatitis, y la enfer- 
Tabla 1. Etiología y prevalencia de la insuficiencia pancreática exocrina en diferentes condiciones clínicas.

\begin{tabular}{|c|c|}
\hline Etiología & Prevalencia \\
\hline \multicolumn{2}{|l|}{ Pancreática } \\
\hline - Pancreatitis crónica & $60 \%-90 \%{ }^{(16)}$ \\
\hline - Pancreatitis aguda & $0 \%-83 \%(2)$ \\
\hline - Pancreatitis autoinmune & $47 \%-76 \%{ }^{(17)}$ \\
\hline - Fibrosis quística & $85 \%{ }^{(7-9)}$ \\
\hline - Diabetes mellitus tipo 1 y 2 & $\begin{array}{l}\text { Tipo 1: } 39 \%{ }^{(18)} \\
\text { Tipo 2: } 28 \%^{(18)}\end{array}$ \\
\hline $\begin{array}{l}\text { - Tumores neuroendocrinos } \\
\text { del páncreas }\end{array}$ & $20 \%{ }^{(19)}$ \\
\hline - Tumores pancreáticos & $60 \%-92 \%$ en cabeza ${ }^{(3)}$ \\
\hline - Cirugía pancreática & $\begin{array}{l}85 \%-95 \% \text { en duodeno- } \\
\text { pancreatectomía cefálica }{ }^{(20)} \\
80 \%-90 \% \text { con } \\
\text { preservación de píloro }{ }^{(20)} \\
20 \%-50 \% \text { en } \\
\text { pancreatectomía distal }^{(21,22)}\end{array}$ \\
\hline \multicolumn{2}{|l|}{ Extrapancreática } \\
\hline $\begin{array}{l}\text { - Síndrome de Shwachman- } \\
\text { Diamond }\end{array}$ & $80 \%-90 \%{ }^{(21)}$ \\
\hline - Enfermedad celíaca & $5 \%-80 \%{ }^{(21)}$ \\
\hline $\begin{array}{l}\text { - Enfermedad inflamatoria } \\
\text { intestinal }\end{array}$ & $\begin{array}{l}10 \% \text { colitis ulcerosa }^{(21)} \\
4 \% \text { enfermedad de } \\
\text { Crohn }^{(21)}\end{array}$ \\
\hline - Cirugía gastrointestinal & $\begin{array}{l}40 \%-80 \% \text { gastrectomía } \\
\text { subtotal/total(21) } \\
31 \% \text { cirugía bariátrica } \\
\text { (bypass gástrico, Y de } \\
\text { Roux) })^{(23)}\end{array}$ \\
\hline $\begin{array}{l}\text { - Virus de inmunodeficiencia } \\
\text { humana }\end{array}$ & $10 \%-50 \%(24)$ \\
\hline - Tabaquismo & $10 \%-18 \%(25)$ \\
\hline
\end{tabular}

Fuente: elaboración propia

medad pancreática previa, como en el caso de la PC subyacente ${ }^{(2)}$.

Una revisión sistemática y metaanálisis realizada por Hollemans y colaboradores ${ }^{(27)}$ incluyó 1495 pacientes, seguidos durante 36 meses del evento inicial. Se identificó que el $25 \%$ de los pacientes desarrollaba IPE durante el seguimiento, y que, en la etiología alcohólica, la PA grave y necrotizante se asociaron con un mayor riesgo de IPE.

En el estudio de Huang y colaboradores ${ }^{(28)}$, en 1795 pacientes con PA se demostró una prevalencia combinada de IPE al ingreso del 62 \% (IC $95 \%$ : 39 \%-82 \%), que disminuye significativamente durante el seguimiento hasta el $35 \%$ (27 \%-43\%; diferencia de riesgo: -0, 34,0,53 a -0,14). La prevalencia de IPE fue mayor en PA grave, en necrosis pancreática y PA de etiología alcohólica. Actualmente se sugiere monitorizar la función pancreática exocrina después de un episodio grave de PA.

\section{Pancreatitis autoinmune}

Es un proceso inflamatorio pancreático que requiere la combinación de datos clínicos, de laboratorio y de imagen. La presentación clínica característica es la ictericia obstructiva, y a menudo se asocia con IPE. En un estudio retrospectivo reciente, la tasa de IPE fue del $47 \%$ y del $76 \%$ en las formas más graves ${ }^{(17)}$.

\section{Fibrosis quística (FQ)}

Es un trastorno autosómico recesivo caracterizado por una mutación en el gen CFTR (cystic fibrosis transmembrane conductance regulator), el cual se expresa en las células ductales pancreáticas ${ }^{(9)}$.

La disfunción del CFTR produce secreciones viscosas deficitarias en agua y bicarbonato, que forman tapones en los ductos y conducen finalmente a la digestión retrógrada de la glándula, con desaparición de los acinos que se reemplazan por tejido fibroso rodeado de zonas quísticas. El $85 \%$ de los pacientes con FQ desarrollará $\mathrm{IPE}^{(7-9)}$.

\section{Diabetes mellitus}

Por definición, la IPE se encuentra en casi todos los pacientes con DM tipo 3C, la cual es secundaria a la PC o al CP. También, tanto en DM tipo 1 como 2, el daño microvascular puede inducir fibrosis pancreática y reducir el volumen pancreático, lo que desencadena la IPE. Los efectos tróficos de la insulina, el control deficiente de la glucemia, la larga duración de la enfermedad, la inflamación y la esteatosis se han asociado con deterioro exocrino ${ }^{(21,23)}$.

La microangiopatía diabética produce isquemia en el páncreas exocrino y la neuropatía autónoma altera los reflejos enteropancreáticos, por ello podrían contribuir al desarrollo de IPE ${ }^{(23)}$. En un metaanálisis de 
17 estudios, que incluyeron a 3662 sujetos con DM, se informó IPE en el $39 \%$ de los pacientes con DM tipo 1 y en el $28 \%$ de los pacientes con DM tipo 2. La DM tipo 3C también se ha asociado con el desarrollo de disfunción exocrina ${ }^{(18)}$.

\section{Tumores neuroendocrinos (TNE)}

Los TNE benignos serosos o mucinosos del páncreas alteran de forma diversa la función exocrina, según el tamaño, la localización, la afectación ductal y la intervención quirúrgica. En los TNE pancreáticos o extrapancreáticos avanzados bien diferenciados se puede observar la IPE moderada en el $20 \%$ de los sujetos tratados con terapia con análogos de somatostatina, debido a la inhibición de la secreción pancreática ${ }^{(19)}$.

\section{Cáncer de páncreas}

ElCP es una neoplasia maligna con mal pronóstico. Es la cuarta causa principal de muerte a nivel mundial ${ }^{(29)}$. El adenocarcinoma ductal de páncreas representa el $90 \%$ de los tumores sólidos malignos de esta glándula; el 10 $\%$ restante incluye a los TNE y a los acinares ${ }^{(2)}$. En los tumores de cabeza de páncreas, la prevalencia de IPE es de $60 \%-70 \%$, y se incrementa a $92 \%$ luego de un promedio de seguimiento de 2 meses $^{(3)}$.

La mayoría de los pacientes con $\mathrm{CP}$ presenta pérdida de peso al momento del diagnóstico, la misma es secundaria al síndrome anorexia-caquexia y a la IPE, que impactan negativamente en el pronóstico de estos pacientes, lo que condiciona el ingreso a los protocolos de tratamiento, $y$ disminuye la calidad de vida y la supervivencia ${ }^{(2,7)}$.

\section{Cirugía pancreática}

Cuando está indicada la cirugía pancreática se debe considerar que la misma altera la anatomía digestiva, la correcta mezcla de los alimentos con la bilis y las enzimas pancreáticas, y reduce el volumen pancreático. Los diferentes procedimientos están asociados con diferentes grados de IPE ${ }^{(21)}$.

El procedimiento de Whipple (duodenopancreatectomía cefálica) determina la tasa más alta de IPE ( $85 \%$ $95 \%)$, pero la misma es ligeramente menor para la intervención con preservación del píloro $(80 \%-90 \%)^{(20)}$. De manera similar, la anastomosis pancreática-yeyunal se asocia con tasas más bajas de IPE en comparación con la gastropancreática ${ }^{(30)}$. La pancreatectomía distal se asocia con una tasa más baja de IPE (20\%-50\%) $)^{(21,22)}$.

\section{Causas extrapancreáticas}

El síndrome de Shwachman-Diamond es un trastorno genético que produce problemas pancreáticos, entre otros. Es la segunda causa hereditaria más común de IPE (entre $80 \%-90 \%)^{(21)}$.

En la enfermedad celíaca no tratada se ha reportado una disminución transitoria de la elastasa fecal (EF-1), con tasas extremadamente heterogéneas (5\%-80\%), que se normaliza con una dieta libre de gluten ${ }^{(21)}$.

En un estudio poblacional muy reciente se demostró una estrecha asociación entre la elastasa pancreática y la diversidad microbiana, lo que subraya la compleja interacción entre el intestino y el páncreas exocrino ${ }^{(23)}$. Se ha notificado afectación pancreática durante el curso de la enfermedad inflamatoria intestinal, con una prevalencia de IPE del $10 \%$ en colitis ulcerosa y $4 \%$ en enfermedad de Crohn ${ }^{(21)}$.

Entre los trastornos infecciosos, la IPE es común en pacientes con virus de inmunodeficiencia humana (VIH), como se confirmó recientemente en un estudio prospectivo que detectó una reducción de EF-1 en pacientes tratados con terapia antirretroviral. La prevalencia de IPE fue entre $10 \%-50 \%{ }^{(24)}$. En un estudio se asoció IPE con TBQ con una prevalencia de $10 \%-18$ $\%$ en sujetos sin enfermedad pancreática ${ }^{(25)}$.

La cirugía gastrointestinal altera la relajación gástrica y la señalización hormonal, lo que afecta negativamente la función exocrina. La IPE se presenta en $40 \%-80 \%$ de los pacientes con resección gástrica total/subtotal, tanto en tumores gástricos como en úlcera péptica ${ }^{(21)}$. La cirugía bariátrica puede ocasionar IPE, el $31 \%$ de los pacientes obesos desarrolló IPE después de la cirugía de bypass gástrico en $\mathrm{Y}$ de Roux ${ }^{(23)}$.

\section{DIAGNÓSTICO DE LA IPE}

El diagnóstico de la IPE se basa en una variedad de pruebas directas o indirectas de la función pancreática. Los métodos directos ofrecen una alta sensibilidad y especificidad, no están fácilmente disponibles, son invasivos, costosos, y requieren mucho tiempo. Por estos motivos no son útiles para el seguimiento postratamiento. Por el contrario, los indirectos carecen de sensibilidad en las etapas iniciales, pero son menos costosos y más fáciles de aplicar. La ausencia de prueba de diagnóstico precisa, disponible y fácilmente reproducible ha contribuido al infradiagnóstico de $\operatorname{IPE}^{(31,32)}$. 
Existe una alta probabilidad de que se pueda establecer un diagnóstico de IPE antes de realizar una prueba de diagnóstico en pacientes con causas definidas de IPE, por ejemplo, PC, PA con necrosis extensa, cáncer de cabeza de páncreas o cirugía gastrointestinal ${ }^{(32)}$.

\section{Prueba de evaluación de la digestión de grasa}

La función pancreática se puede evaluar mediante el coeficiente de absorción de grasas (CFA) y la prueba de aliento con triglicéridos mixtos marcados en el aire exhalado ${ }^{13} \mathrm{C}\left({ }^{13} \mathrm{C}-\mathrm{MTG}\right)$.

El CFA no es específico de IPE, ni se puede aplicar fácilmente en la práctica clínica. Los pacientes deben ingerir una dieta con $100 \mathrm{~g}$ de grasa durante 5 días consecutivos y recolectar toda la materia fecal de los últimos 3 días ${ }^{(3,15)}$.

La prueba de aliento es un método que consiste en administrar triglicéridos marcados con $\mathrm{C}^{13}\left({ }^{13} \mathrm{C}\right.$ MTG), que se digieren por la lipasa pancreática, y luego se absorben y metabolizan. Después de esto, el $\mathrm{C}^{13}$ se incorpora en forma de ${ }^{13} \mathrm{CO}_{2}$ y se elimina a través del aire espirado. Es una prueba simple, no invasiva y precisa para el diagnóstico de IPE y la evaluación de la eficacia de la TREP en la práctica clínica, pero aún no está ampliamente disponible ${ }^{(2,4)}$.

\section{Prueba de evaluación de la secreción pancreática}

La secreción pancreática puede evaluarse mediante la prueba endoscópica de función pancreática y la cuantificación de la concentración de EF-1 ${ }^{(3)}$. La prueba EF-1 es la más utilizada en la práctica clínica. Es fácil de realizar y se encuentra ampliamente disponible, pero su precisión para la IPE es limitada ${ }^{(15)}$. Los valores de EF-1 menores de $200 \mu \mathrm{g} / \mathrm{g}$ se asocian con IPE, mientras que valores mayores de $500 \mu \mathrm{g} / \mathrm{g}$ permiten excluirla ${ }^{(3,32)}$.

\section{Prueba de Van de Kamer/esteatocrito}

Su uso se ha limitado por tener una forma de realizarse dificultosa y la escasa aceptación de los pacientes ${ }^{(2)}$.

\section{Otros métodos diagnósticos de IPE}

Parámetros nutricionales: clínicos, antropométricos, bioquímicos y funcionales.

La elección del método dependerá del escenario en el que deba diagnosticar la IPE y de la disponibilidad de las pruebas ${ }^{(2)}$.
En la actualidad se sugiere que el correcto diagnóstico de IPE debe basarse en la evaluación combinada de síntomas relacionados con mala digestión, malabsorción de nutrientes, parámetros nutricionales y la prueba de EF-1 en el contexto clínico apropiado ${ }^{(3,32)}$. La combinación de dos de estos tres criterios debe considerarse suficiente para el diagnóstico y para iniciar el tratamiento de la mala digestión pancreática (Figura 2).

\section{EVALUACIÓN NUTRICIONAL (EN)}

El proceso de atención nutricional debe formar parte de la atención integral de los pacientes con IPE. El estado nutricional en pacientes con IPE puede variar desde obesidad a DN severa, por tal motivo, la EN debe realizarse desde el momento del diagnóstico y durante todo el seguimiento, con la finalidad de determinar el diagnóstico nutricional y planificar una intervención nutricional adecuada y oportuna ${ }^{(15,33,34)}$.

En la literatura podemos encontrar varias herramientas de tamizaje nutricional para utilizar en el entorno clínico o ambulatorio, que permitirán identificar a los sujetos con riesgo nutricional ${ }^{(35,36)}$. La herramienta más adecuada es aquella que sea fácil de aplicar en la práctica clínica, sensible a los cambios agudos y crónicos del estado nutricional, no invasiva, validada y específica. La elección dependerá de cada institución ${ }^{(37)}$.

En los pacientes que presentan riesgo de $\mathrm{DN}$ se deberá aplicar una EN formal y extensa para determinar el diagnóstico nutricional ${ }^{(38)}$. La EN completa deberá ser aplicada por un nutricionista debidamente especializado, que disponga del equipamiento necesario para tal fin y debe incluir, además, la combinación de los siguientes parámetros $(\text { Tabla } 2)^{(5,15,37)}$ :

- Parámetros clínicos: consiste en la evaluación de los síntomas que impactan negativamente en la ingesta oral (náuseas, dolor, saciedad precoz, esteatorrea, hinchazón, flatulencia, entre otros), anamnesis alimentaria completa, evaluación de tipo de alimentación tolerada, cálculo de ingesta calórica-proteica y determinación del porcentaje de los requerimientos nutricionales que recibe. También evaluar cambios en el apetito, en los niveles de actividad física y el abandono de hábitos tóxicos (tabaco, alcohol $)^{(1,5,10)}$

- Parámetros antropométricos: peso actual (PAc) y habitual $(\mathrm{PH})$, talla, porcentaje de pérdida de peso (\% PP), índice de masa corporal (IMC), pliegues cutáneos y circunferencias. El peso y el IMC tradicionalmente se han utilizado para diagnosticar DN, pero el IMC no debe utilizarse de forma aislada 


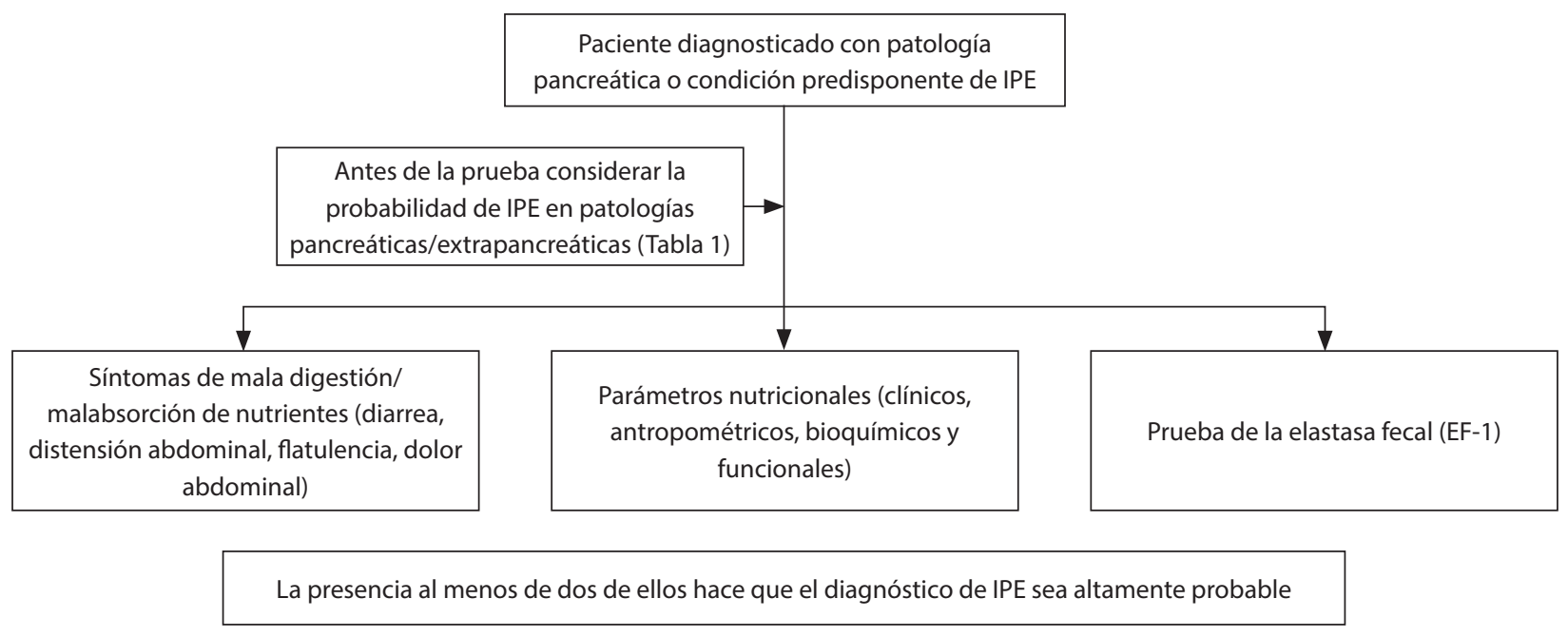

Figura 2. Algoritmo de diagnóstico de insuficiencia pancreática exocrina en la práctica clínica. Adaptado de ${ }^{(4)}$.

Tabla 2. Evaluación nutricional completa en pacientes con insuficiencia pancreática exocrina

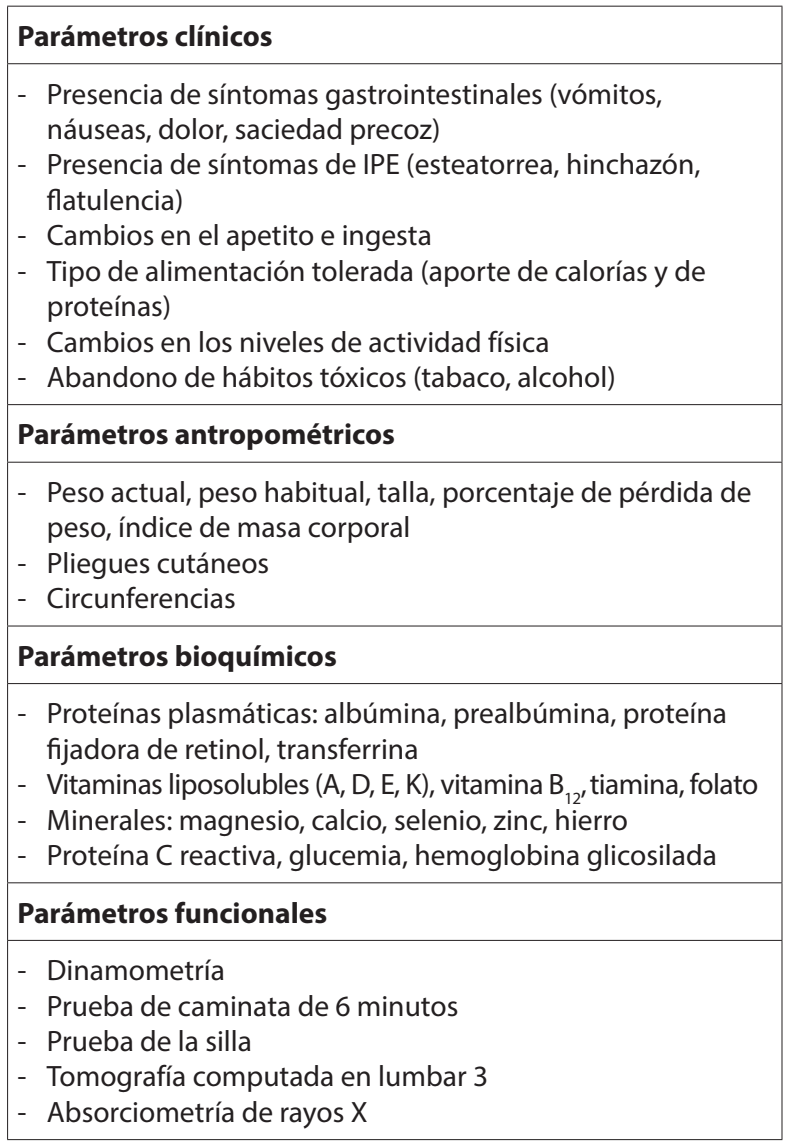

IPE: insuficiencia pancreática exocrina. Fuente: elaboración propia debido a que puede encontrarse aumentado en pacientes con PP. El \% PP se refiere a una pérdida involuntaria, y relaciona la variación entre el $\mathrm{PH}$ y el PAc en función del tiempo. Es considerado el parámetro más significativo como marcador del inicio de $\mathrm{DN}$ en pacientes con PC, y se asocia con aumento de riesgo en pacientes quirúrgicos ${ }^{(3,5,11)}$.

- Parámetros bioquímicos: permiten determinar deficiencias nutricionales. Es recomendable evaluar la concentración de proteínas plasmáticas y de vitaminas liposolubles, ya que su déficit es común en pacientes con IPE, el déficit de vitamina $B_{12}$ yla tiamina en PC de origen alcohólico. Los micronutrientes que habitualmente están comprometidos en IPE son el magnesio, calcio, selenio, hierro y zinc. El estado inflamatorio se debe evaluar mediante la proteína C-reactiva. Valorar también niveles de glucemia y hemoglobina glicosilada, para controlar la correcta función endocrina y el desarrollo de DM tipo $3 \mathrm{C}^{(10,15)}$.

- Pruebas funcionales: permiten determinar sarcopenia mediante la evaluación de la fuerza y la masa muscular, y el rendimiento físico. La dinamometría es una medida simple de la fuerza muscular, y puede correlacionarse con el diagnóstico o pronóstico nutricional ${ }^{(10,11,14)}$. Otros métodos pueden ser la prueba de caminata de 6 minutos o la prueba de la silla ${ }^{(14)}$. Actualmente existen estudios más sofisticados para evaluar la composición corporal, que se consideran los métodos de referencia, como la tomografía computada a nivel de lumbar $3^{(5,10,15)}$. 
El examen de densidad ósea, también denominada absorciometría de rayos $\mathrm{X}$ de energía dual, es la herramienta para identificar pacientes con PC con osteopatía ${ }^{(11)}$. Se debe realizar cada 2 años en aquellos pacientes con osteopenia $^{(5)}$. El resultado de la EN debe documentarse en la historia clínica para que todo el equipo de salud conozca el diagnóstico nutricional del paciente y el plan de intervención que se implementará( ${ }^{(37)}$.

\section{TRATAMIENTO DE LA IPE}

El tratamiento de IPE tiene el objetivo de lograr la correcta digesto-absorción de los alimentos, controlar los síntomas y mejorar el estado nutricional de los pacientes. Consiste en la indicación de TREP junto con un asesoramiento nutricional individualizado respecto a las características de las cápsulas y al plan de alimentación ${ }^{(2)}$.

La dosis de enzimas al comienzo es baja y debe incrementarse según el contenido de grasa de la dieta, las características de las heces y los síntomas de cada paciente. Debe administrarse una dosis capaz de evitar los síntomas gastrointestinales y normalizar el estado nutricional ${ }^{(2,11)}$.

Se sugiere una dosis inicial de 40000 a 50000 unidades internacionales (UI) de lipasa con las comidas principales, y la mitad de esa dosis en las comidas menores. Esta dosis puede ser insuficiente en pacientes con CP, quienes pueden requerir una dosis de $75000 \mathrm{UI}$ por $\operatorname{comida}^{(2,5)}$.

La TREP debe imitar la fisiología pancreática, tanto como sea posible, a través de microesferas con recubrimiento entérico o microesferas de menos de $2 \mathrm{~mm}$ de tamaño ${ }^{(11)}$. El recubrimiento entérico evita la inactivación de las enzimas en el estómago, que requiere un $\mathrm{pH}$ mayor de 5,5 para que se disuelva ${ }^{(31)}$. Por eso, algunos pacientes se benefician de la adición de un inhibidor de bomba de protones (IBP) $)^{(4,7,11)}$. En cuanto a la intervención nutricional, la misma estará adaptada al esquema enzimático indicado.

\section{Tratamiento nutricional}

Se indicará un plan de alimentación individualizado, saludable y con cambios en el estilo de vida (suspensión de hábitos tóxicos) ${ }^{(2,4)}$. Históricamente, las dietas bajas en grasas eran indicadas con el objetivo de disminuir la esteatorrea. Luego se observó que esta práctica agravaba la pérdida de peso, enmascaraba la esteatorrea y empeoraba la deficiencia de vitaminas liposolubles ${ }^{(7,}$ ${ }^{15,31)}$. Varios estudios demostraron que $30 \%-33 \%$ de la energía en forma de grasa es bien tolerada en pacientes con $\operatorname{IPE}^{(5,7)}$.

La restricción de grasas en la dieta solo se recomienda como último recurso para controlar la esteatorrea sintomática en sujetos en los que los ajustes de dosis en la TREP y la adición de IBP no han dado como resultado un alivio satisfactorio de los síntomas ${ }^{(5)}$.

En pacientes con una secreción pancreática reducida es preferible el consumo de comidas pequeñas, frecuentes y con alto contenido energético ${ }^{(7,11,39)}$. El aporte calórico y proteico dependerá del estado nutricional. Se estima entre $25-35 \mathrm{kcal} / \mathrm{kg}$ de peso corporal/día y $1,2-1,5 \mathrm{~g} / \mathrm{kg}$ de peso corporal/día de proteína ${ }^{(5,7,40)}$. La selección de carbohidratos dependerá del control glucémico. En particular, no se dispone de recomendaciones dietéticas específicas para pacientes con DM e IPE ${ }^{(22)}$.

En la literatura se puede encontrar la recomendación de evitar dietas con elevado aporte de fibra (75-80 g de fibra por día $)^{(41)}$, ya que afectaría negativamente en la acción de las enzimas pancreáticas. Sin embargo, una dieta con la recomendación dietética adecuada de fibra, considerando las condiciones clínicas individuales, no traería efectos adversos ${ }^{(3,5,11,37,42)}$. La suplementación con vitaminas liposolubles, hidrosolubles y micronutrientes debe considerarse en pacientes con bajas concentraciones plasmáticas ${ }^{(11)}$.

\section{TERAPIA NUTRICIONAL (TN)}

Cuando los pacientes no puedan cubrir requerimientos nutricionales se sugiere iniciar TN mediante suplementos nutricionales orales (SNO), nutrición enteral (NE) o nutrición parenteral (NP). Su principal objetivo es prevenir o tratar la $\mathrm{DN}^{(5,11,15,37)}$.

\section{Terapia nutricional oral}

En un estudio controlado aleatorizado se compararon los consejos dietéticos y TN con SNO en 1 cohorte de 60 pacientes con $\mathrm{DN}$ y con PC. Los pacientes con intervención nutricional presentaron descenso del dolor $\mathrm{y}$ mejoría del estado nutricional ${ }^{(7,43)}$.

\section{Nutrición enteral}

Estará indicada cuando la vía oral (VO) resulte insuficiente. Se recomienda utilizar fórmulas oligoméricas o semielementales, que contienen triglicéridos de cadena media (TCM), los cuáles no requieren lipasa pancreática para su absorción. En caso de no disponer de este 
tipo de fórmulas se pueden utilizar las fórmulas poliméricas, pero considere que el paciente con IPE no podrá absorber adecuadamente ${ }^{(11)}$. Una alternativa de $\mathrm{NE}$ complementaria puede ser NE cíclica nocturna con fórmula oligomérica y la TREP se deberá calcular en función de la VO.

La administración de enzimas pancreáticas mediante la fórmula de NE no está respaldada por directrices, genera mayor manipulación e incluso puede obstruir la sonda de alimentación. Además, no existe un estudio prospectivo que demuestre la eficacia o seguridad de esta práctica ${ }^{(37,44)}$.

\section{Nutrición parenteral}

Se indicará cuando la vía digestiva se encuentra inhabilitada. No requiere el uso de enzimas pancreáticas.

\section{¿Cómo controlar la eficacia de TREP?}

Para garantizar una óptima eficacia de la TREP se deberá evaluar:

- El alivio de síntomas gastrointestinales y la mejora de parámetros nutricionales ${ }^{(2,11)}$.

- La dosis de enzimas debe estar ajustada a la cantidad de grasa que consumen los pacientes ${ }^{(15,37)}$.

- Las cápsulas deben ingerirse y tragarse intactas, sin triturar ni masticar, con suficiente cantidad de líquido y durante las comidas. Para comidas de larga duración distribuir las cápsulas ${ }^{(5,15,37)}$.

- Confirmar que los pacientes ingieren las enzimas correctamente en momento y cantidad ${ }^{(2,15,33)}$.

- Considerar el abandono de hábitos tóxicos $(\mathrm{TBQ}$ alcohol) $)^{(2)}$.

- En caso de una respuesta tórpida al tratamiento se podrá necesitar ajustar la dosis enzimática, indicar un IBP, restringir las grasas y aportar TCM. Considerar la posibilidad de diagnósticos alternativos (sobrecrecimiento bacteriano, entre otros) ${ }^{(2)}$.

\section{CONCLUSIÓN}

La IPE es una afección clínica importante y a menudo no diagnosticada, con posibles efectos deletéreos sobre el estado nutricional de los pacientes con trastornos pancreáticos y extrapancreáticos.

El diagnóstico y el tratamiento de la IPE es subóptimo, lo que subraya la importancia de la concientización en pacientes de alto riesgo, uso apropiado de las pruebas de diagnóstico y comprensión de los objetivos y estrategias del tratamiento.
La TREP es la piedra angular del tratamiento de la IPE. Dentro de las estrategias del tratamiento nutricional se debe evaluar al paciente con parámetros clínicos, antropométricos, bioquímicos y funcionales. En caso de que con la $\mathrm{VO}$ no pueda cubrir los requerimientos nutricionales, se sugiere iniciar la TN para mejorar el estado nutricional. El seguimiento de los pacientes debe estar acompañado por la evaluación periódica de un nutricionista, que debe formar parte del equipo multidisciplinario.

\section{Financiamiento}

El presente estudio no tuvo financiación.

\section{Conflicto de intereses}

Los autores declaran no tener conflicto de intereses.

\section{Declaración de autoría}

$\mathrm{MC}, \mathrm{LDN}$ y AV contribuyeron igualmente a la concepción y diseño de la investigación; MC, LDN y AV redactaron el manuscrito. Todos los autores revisaron el manuscrito, acuerdan ser plenamente responsables de garantizar la integridad y precisión del trabajo, y leyeron $y$ aprobaron el manuscrito final.

\section{Referencias bibliográficas}

1. Othman MO, Harb D, Barkin JA. Introduction and practical approach to exocrine pancreatic insufficiency for the practicing clinician. Int J Clin Pract. 2018;72(2):e13066. doi: 10.1111/ijcp.13066.

2. Basso S, Canicoba M, Capitanich P, Carballido M, Crisci J, Giustina FD, et al. I Consenso argentino de diagnóstico y tratamiento de la insuficiencia pancreática exocrina. Acta Gastroentero Latinoam. 2018;48(3):213-25.

3. Dominguez-Muñoz JE. Management of pancreatic exocrine insufficiency. Curr Opin Gastroenterol. 2019;35(5):455-9. doi: 10.1097/MOG.0000000000000562.

4. Dominguez-Muñoz JE. Diagnosis and treatment of pancreatic exocrine insufficiency. Curr Opin Gastroenterol. 2018;34(5):349-54. doi: 10.1097/ MOG.0000000000000459.

5. Löhr JM, Dominguez-Munoz E, Rosendahl J, Besselink M, Mayerle J, Lerch M, et al. United European Gastroenterology evidence-based guidelines for the diagnosis and therapy of chronic pancreatitis (HaPanEU). United European Gastroenterol J. 2017;5(2):153-99. doi: 10.1177/2050640616684695.

6. Layer P, Kashirskaya N, Gubergrits N. Contribution of pancreatic enzyme replacement therapy to survival and quality of life in patients with pancreatic exocrine insufficiency. World J 
Gastroenterol. 2019;25(20):2430-41. doi: 10.3748/wjg.v25. i20.2430.

7. Gheorghe C, Seicean A, Saftoiu A, Tantau M, Dumitru E, Jinga $\mathrm{M}$, et al. Romanian guidelines on the diagnosis and treatment of exocrine pancreatic insufficiency.J Gastrointestin Liver Dis. 2015;24(1):117-23. doi: 10.15403/jgld.2014.1121.app.

8. Working Party of the Australasian Pancreatic Club, Smith RC, Smith SF, Wilson J, Pearce C, Wray N, et al. Summary and recommendations from the Australasian guidelines for the management of pancreatic exocrine insufficiency. Pancreatology. 2016;16(2):164-80. doi: 10.1016/j. pan.2015.12.006.

9. Perbtani Y, Forsmark CE. Update on the diagnosis and management of exocrine pancreatic insufficiency. F1000Res. 2019;8:F1000. doi: 10.12688/f1000research.20779.1.

10. Lindkvist B, Phillips ME, Domínguez-Muñoz JE. Clinical, anthropometric and laboratory nutritional markers of pancreatic exocrine insufficiency: Prevalence and diagnostic use. Pancreatology. 2015;15(6):589-97. doi: 10.1016/j. pan.2015.07.001.

11. Arvanitakis M, Ockenga J, Bezmarevic M, Gianotti L, Krznarić $\check{Z}$, Lobo DN, et al. ESPEN guideline on clinical nutrition in acute and chronic pancreatitis. Clin Nutr. 2020;39(3):612-31. doi: 10.1016/j.clnu.2020.01.004.

12. Duggan SN, Smyth ND, Murphy A, Macnaughton D, O'Keefe SJD, Conlon KC. High prevalence of osteoporosis in patients with chronic pancreatitis: A systematic review and metaanalysis. Clin Gastroenterol Hepatol. 2014;12(2):219-28. doi: $10.1016 /$ j.cgh.2013.06.016.

13. De la Iglesia D, Vallejo-Senra N, López-López A, IglesiasGarcía J, Lariño-Noia J, Nieto-García L, et al . Pancreatic exocrine insufficiency and cardiovascular risk in patients with chronic pancreatitis: A prospective, longitudinal cohort study. J Gastroenterol Hepatol. 2019;34(1):277-83. doi: 10.1111/ jgh.14460.

14. Shintakuya R, Uemura K, Murakami Y, Kondo N, Nakagawa $\mathrm{N}$, Urabe K, et al. Sarcopenia is closely associated with pancreatic exocrine insufficiency in patients with pancreatic disease. Pancreatology. 2017;17(1):70-5. doi: 10.1016/j. pan.2016.10.005.

15. Domínguez-Muñoz JE, Phillips M. Nutritional therapy in chronic pancreatitis. Gastroenterol Clin North Am. 2018;47(1):95-106. doi: 10.1016/j.gtc.2017.09.004.

16. Machicado JD, Chari ST, Timmons L, Tang G, Yadav D. A population-based evaluation of the natural history of chronic pancreatitis. Pancreatology. 2018;18(1):39-45. doi: 10.1016/j.pan.2017.11.012.

17. Vujasinovic M, Valente R, Maier P, von Beckerath V, Haas SL, Arnelo U, et al. Diagnosis, treatment and long-term outcome of autoimmune pancreatitis in Sweden. Pancreatology. 2018;18(8):900-4. doi: 10.1016/j.pan.2018.09.003.

18. Mohapatra S, Majumder S, Smyrk TC, Zhang L, Matveyenko A, Kudva YC, et al. Diabetes mellitus is associated with an exocrine pancreatopathy: Conclusions from a review of literature. Pancreas. 2016;45(8):1104-10. doi: 10.1097/ MPA.0000000000000609.

19. Lamarca A, McCallum L, Nuttall C, Barriuso J, Backen A, Frizziero $\mathrm{M}$, et al. Somatostatin analogue-induced pancreatic exocrine insufficiency in patients with neuroendocrine tumors: Results of a prospective observational study. Expert Rev Gastroenterol Hepatol. 2018;12(7):723-31. doi: 10.1080/17474124.2018.1489232.

20. Goess R, Ceyhan GO, Friess H. Pancreatic exocrine insufficiency after pancreatic surgery. Panminerva Med. 2016;58(2):151-9.

21. Capurso G, Traini M, Piciucchi M, Signoretti M, Arcidiacono PG. Exocrine pancreatic insufficiency: Prevalence, diagnosis, and management. Clin Exp Gastroenterol. 2019;12:129-39. doi: 10.2147/CEG.S168266.

22. Okano K, Murakami Y, Nakagawa N, Uemura K, Sudo T, Hashimoto $Y$, et al. Remnant pancreatic parenchymal volume predicts postoperative pancreatic exocrine insufficiency after pancreatectomy. Surgery. 2016;159(3):885-92. doi: 10.1016/j.surg.2015.08.046.

23. Radlinger B, Ramoser G, Kaser S. Exocrine pancreatic insufficiency in type 1 and type 2 diabetes. Curr Diab Rep. 2020;20(6):18. doi: 10.1007/s11892-020-01304-0.

24. Yilmaz A, Hagberg L. Exocrine pancreatic insufficiency is common in people living with HIV on effective antiretroviral therapy. Infect Dis (Lond). 2018;50(3):193-9. doi: 10.1080/23744235.2017.1370126.

25. Raphael KL, Chawla S, Kim S, Keith CG, Propp DR, Chen $\mathrm{ZN}$, et al. Pancreatic insufficiency secondary to tobacco exposure: A controlled cross-sectional evaluation. Pancreas. 2017;46(2):237-43. doi: 10.1097/MPA.0000000000000721.

26. Greer JB, Greer P, Sandhu BS, Alkaade S, Wilcox CM, Anderson MA, et al. Nutrition and inflammatory biomarkers in chronic pancreatitis patients. Nutr Clin Pract. 2019; 34(3):387-99. doi: 10.1002/ncp.10186.

27. Hollemans RA, Hallensleben NDL, Mager DJ, Kelder JC, Besselink MG, Bruno MJ, et al. Pancreatic exocrine insufficiency following acute pancreatitis: Systematic review and study level meta-analysis. Pancreatology. 2018;18(3):253-62. doi: 10.1016/j.pan.2018.02.009.

28. Huang W, De la Iglesia-García D, Baston-Rey I, CalviñoSuarez C, Lariño-Noia J, Iglesias-García J, et al. Exocrine pancreatic insufficiency following acute pancreatitis: Systematic review and meta-analysis. Dig Dis Sci. 2019;64(7):19852005. doi: 10.1007/s10620-019-05568-9.

29. De la Iglesia D, Avci B, Kiriukova M, Panic N, Bozhychko M, Sandru V, et al. Pancreatic exocrine insufficiency and pancreatic enzyme replacement therapy in patients with advanced pancreatic cancer: A systematic review and meta-analysis. United European Gastroenterol J. 2020;8(9):1115-25. doi: $10.1177 / 2050640620938987$.

30. Roeyen G, Jansen M, Ruyssinck L, Chapelle T, Vanlander A, Bracke B, et al. Pancreatic exocrine insufficiency after pancreaticoduodenectomy is more prevalent with pancreatico- 
gastrostomy than with pancreatic jejunostomy. A retrospective multicentre observational cohort study. HPB (Oxford). 2016;18(12):1017-22. doi: 10.1016/j.hpb.2016.09.002.

31. Struyvenberg MR, Martin CR, Freedman SD. Practical guide to exocrine pancreatic insufficiency - Breaking the myths. BMC Med. 2017;15(1):29. doi: 10.1186/s12916-0170783-y.

32. Diéguez-Castillo C, Jiménez-Luna C, Prados J, Martín-Ruiz JL, Caba O. State of the art in exocrine pancreatic insufficiency. Medicina (Kaunas). 2020;56(10):523. doi: 10.3390/ medicina56100523.

33. Rodrigues-Pinto E, Caldeira A, Soares JB, Antunes T, Carvalho JR, Costa- Maia J,et al. Clube português do pâncreas recommendations for chronic pancreatitis: medical, endoscopic, and surgical treatment (Part II). GE Port J Gastroenterol. 2019; 26(6):404-13. doi: 10.1159/000497389.

34. Canicoba ME, Mauricio S. Valoración del estado nutricional en diversas situaciones clínicas. Los Olivos, Lima: Universidad Privada del Norte. 2017.

35. Cortes R, Bennasar-Veny M, Castro-Sanchez E, Fresneda $S$, de Pedro-Gomez J, Yañez A. Nutrition screening tools for risk of malnutrition among hospitalized patients: A protocol for systematic review and meta-analysis. Medicine (Baltimore). 2020;99(43):e22601. doi: 10.1097/ MD.0000000000022601.

36. Di Sibio M, Jastreblansky Z, Magnífico LP, Fischberg M, Ramírez SE, Jereb S, et al. Revisión de diferentes herramientas de tamizaje nutricional para pacientes hospitalizados. Diaeta. 2018; 36(164):30-38.

37. Moro, M, Kohan G, Pasqua A, Volpacchio M, Marcaccio, F, Kujaruk M, et al. Recomendaciones para el diagnóstico y tratamiento del adenocarcinoma ductal de páncreas. Acta Gastroenterol Latinoam. 2020;50(S2):1-43.

38. Lobo DN, Gianotti L, Adiamah A, Barazzoni R, Deutz NEP, Dhatariya $\mathrm{K}$, et al. Perioperative nutrition: Recommendations from the ESPEN expert group. Clin Nutr. 2020;39(11):321127. doi: 10.1016/j.clnu.2020.03.038.

39. Dominguez-Munoz JE, Drewes AM, Lindkvist B, Ewald N, Czakó L, RosendahlJ, et al. Recommendations from the United European Gastroenterology evidence-based guidelines for the diagnosis and therapy of chronic pancreatitis. Pancreatology. 2018;18(8):847-54. doi: 10.1016/j.pan.2018.09.016.

40. Duggan S, O'Sullivan M, Feehan S, Ridgway P, Conlon K. Nutrition treatment of deficiency and malnutrition in chronic pancreatitis: A review. Nutr Clin Pract. 2010;25(4):362-70. doi: $10.1177 / 0884533610373772$.

41. Dutta SK, Hlasko J. Dietary fiber in pancreatic disease: Effect of high fiber diet on fat malabsorption in pancreatic insufficiency and in vitro study of the interaction of dietary fiber with pancreatic enzymes. Am J Clin Nutr. 1985;41(3):51725. doi: 10.1093/ajen/41.3.517.

42. Ribichini E, Stigliano S, Rossi S, Zaccari P, Sacchi MC, Bruno $\mathrm{G}$, et al. Role of fibre in nutritional management of pancreatic diseases. Nutrients. 2019;11(9):2219. doi: 10.3390/ nu11092219.

43. Singh S, Midha S, Singh N, Joshi YK, Garg PK. Dietary counseling versus dietary supplements for malnutrition in chronic pancreatitis: A randomized controlled trial. Clin Gastroenterol Hepatol. 2008;6(3):353-9. doi: 10.1016/j.cgh.2007.12.040.

44. Freedman SD. Options for addressing exocrine pancreatic insufficiency in patients receiving enteral nutrition supplementation. Am J Manag Care. 2017;23(12):S220-8. 